Rizwan Khan, MD

Department of Critical Care Medicine,

Respiratory Institute, Heart, Vascular,

and Thoracic Institute, Cleveland Clinic
Balaram Anandamurthy, MD

Department of Cardiothoracic Anesthesiology,

Heart, Vascular, and Thoracic Institute,

Cleveland Clinic
Kenneth McCurry, MD

Department of Thoracic and Cardiovascular

Surgery, Heart, Vascular, and Thoracic Institute,

Cleveland Clinic
Sudhir Krishnan, MD

Department of Critical Care Medicine,

Respiratory Institute, Heart, Vascular,

and Thoracic Institute, Cleveland Clinic

\title{
Utility of extracorporeal membrane oxygenation in COVID-19
}

\section{Posted April 28, 2020}

\section{ABSTRACT}

Extracorporeal membrane oxygenation (ECMO) is a means of cardiopulmonary support for refractory respiratory and cardiac failure. ECMO is a resource-intensive therapy that can be considered in highly selected patients. Expert centers should employ an evidence-based ARDS treatment algorithm and a multidisciplinary approach to recommending ECMO upon failure of conventional therapy. Caring for ECMO patients requires adequate infection control and safety precautions for healthcare workers.

\section{INTRODUCTION}

As the coronavirus disease 2019 (COVID-19) pandemic rages, we expect to see an increase in the number of patients receiving extracorporeal membrane oxygenation (ECMO) This poses a dilemma, as ECMO is expensive and labor-intensive, access to it is limited, and clinicians need to be highly selective in using it.

\section{WHAT IS ECMO?}

ECMO is a means of cardiopulmonary support for refractory respiratory and cardiac failure. There are 2 main types: venovenous and venoarterial.

Venovenous ECMO is used to correct hypoxemia and hypercarbia. In one form, a single dual-lumen catheter draws blood from a large vein and reinfuses oxygenated blood back into the right atrium. Another uses 2 cannulas, usually drawing blood from a femoral vein and reinfusing it via the internal jugular vein into the right atrium. Dual-site cannulation is preferred for COVID-19 patients for pulmonary support, as it can be performed safely at the bedside without the need

The statements and opinions expressed in COVID-19 Curbside Consults are based on experience and the available literature as of the date posted. While we try to regularly update this content, any offered recommendations cannot be substituted for the clinical judgment of clinicians caring for individual patients.

doi:10.3949/ccjm.87a.ccc014 for fluoroscopy or transesophageal echocardiography, so that fewer personnel need to come into the room.

Venoarterial ECMO provides cardiac and circulatory support in addition to oxygenation.

\section{THE ONLY RECOURSE IF CONVENTIONAL ARDS TREATMENT FAILS}

COVID-19 caused by severe acute respiratory syndrome coronavirus 2 (SARS-CoV-2) was declared a pandemic by the World Health Organization (WHO) on March 11, 2020. COVID-19 causes severe acute respiratory distress syndrome (ARDS) as well as myocardial depression secondary to acute cardiac injury in a subset of patients. Advanced age and medical comorbidities have been identified as risk factors for poor outcome. Fifteen percent of patients could have severe disease warranting hospital admission, and $5 \%$ are designated as critically ill. ${ }^{1}$

ARDS is a form of diffuse inflammatory lung injury that leads to increased vascular permeability, culminating in hypoxemic respiratory failure. Histopathologic study of COVID-19-induced lung injury reveals diffuse alveolar damage, desquamation of pneumocytes, and pulmonary edema with hyaline membrane formation consistent with ARDS. ${ }^{2}$ Severe ARDS is usually managed conventionally with mechanical ventilation (using a lung-protective strategy), adjuvant neuromuscular blockade, and prone ventilation, depending on the severity of the hypoxemia.

ECMO is the only recourse available if conventional therapy fails. It permits ultra-lung-protective strategies on mechanical ventilation, avoiding ventilator-induced lung injury while allowing the pulmonary parenchyma and function to recover with time.

Nonrandomized studies, case series, and reports of using ECMO as a bridge to recovery in ARDS during the 2009 influenza A (H1N1) epidemic suggest that the mortality rate is lower with ECMO. ${ }^{3}$ Similarly, a lower mortality rate was reported for patients with refractory hypoxemia undergoing ECMO therapy 
during the Middle East respiratory syndrome coronavirus (MERS-CoV) epidemic in Saudi Arabia in 2014. ${ }^{4}$ However, in the ECMO to Rescue Lung Injury in Severe ARDS (EOLIA) trial, the largest trial to date of early use of ECMO in severe ARDS, Coombes et al reported a statistically insignificant $11 \%$ absolute reduction in mortality at 60 days. ${ }^{5}$

Evolution in ECMO technology and increasing medical evidence attest to the feasibility and safety of this therapy in ARDS and permit its consideration in this current pandemic. Nonetheless, the role of ECMO in the management of COVID-19 is not clearly defined. In a recent report from China, 14 (82\%) of 17 ARDS patients receiving ECMO as a rescue strategy died. ${ }^{6}$ A report from Italy ${ }^{7}$ found that only $1 \%$ of eligible patients were placed on ECMO in the first 24 hours of presentation. Some of the patients were still on ECMO at the time of publication of these articles, limiting the interpretability of these studies. However, early reports from Japan are encouraging, with 21 of 40 patients reported to recover on ECMO support. ${ }^{8}$

\section{DRAWBACKS OF ECMO}

Limited role as cardiac support. Acute cardiac injury from COVID-19, though less common than ARDS, still occurs in about $20 \%$ of patients. ${ }^{9}$ It manifests as myocarditis, severe left ventricular dysfunction, and cardiac arrhythmias. The role of venoarterial ECMO in cardiogenic shock is limited in these patients due to complexities of patient management and increased mortality. There are only a few reports of successful use of venoarterial ECMO in myocarditis secondary to COVID-19.10 Use of venoarterial ECMO should be based on stringent criteria and subject to scrutiny by ECMO multidisciplinary teams.

ECMO and thrombosis. Starting ECMO initiates the coagulation cascade, necessitating systemic anticoagulation. Moreover, COVID-19 itself is associated with systemic thrombotic complications, adding to this clinical conundrum and possibly increasing the propensity for thrombosis and failure of the oxygenator membrane. ${ }^{11}$ However, as yet, there is no evidence to support a change in the recommended anticoagulant strategy for COVID-19 patients on EMCO.

Logistic challenges. Providing ECMO during a global pandemic poses ethical and logistical challenges. ECMO is provided only at expert centers due to its high operational cost and need for a specialist multidisciplinary team. Expert centers have expert personnel and advanced therapeutic interventions to support patients on ECMO and the expertise to tackle ECMO-associated complications.
WHO interim guidelines recommend referring patients with refractory hypoxemia to expert centers capable of providing ECMO for COVID-19-associated ARDS. ${ }^{12}$ Additionally, the Extracorporeal Life Support Organization ${ }^{13}$ recommends that ECMO for COVID-19-related disease be done at existing and experienced extracorporeal life support centers, and recommends against setting up new ECMO centers for the sole purpose of treating COVID-19 patients.

ECMO and limited medical resources. The rapid consumption of healthcare resources in the current epidemic has overwhelmed healthcare systems throughout the world. In times of crisis, if resources are in short supply, offering prolonged ECMO, even if medically indicated, could mean diverting resources from other critically ill patients. In pandemic situations it is incumbent upon healthcare systems to reserve resources and personnel for contingency and crises (ie, "surge" capacity) to be able to support an ECMO program. Training and involvement of additional healthcare personnel at all levels to ensure adequate reserve capacity and continued provision of ECMO service due to the loss of healthcare personnel to the pandemic also needs to be accounted for.

Risk of infection in caregivers. SARS-COV-2 is highly transmissible, and ECMO demands an inordinate amount of maintenance, exposing the ECMO specialists and other caregivers to higher risks. Shortages of personal protective equipment that could develop in a crisis could compromise the safety of healthcare workers.

Limited utility in multiorgan failure. Emerging data indicate that the virus causes multiorgan failure through hypoxemia and shock. The utility of ECMO in patients with multiorgan failure is limited, with an abysmal return for the investment of resources.

\section{TRIAGE FOR ECMO}

Priority is given to patients who are younger, without comorbidities, likely to need ECMO for only a short time, and most likely to survive. Clustering eligible patients (with severe ARDS or early transfer) at expert centers where ECMO resources are readily available is clinically and logistically prudent. Indications for and contraindications to ECMO in COVID19 patients at our center are listed in Table 1 .

Patients should be triaged (sorted, classified, and assigned) to ECMO therapy based on primary criteria (ie, highest likelihood of survival and short-term need for ECMO) and secondary criteria (expected duration of life and expected quality of life after ECMO therapy). There are no published studies or guidelines that list selection criteria or duration of extracorporeal 
life support therapy specifically for COVID-19-associated ARDS. Recommendations are extrapolated from data obtained during the MERS, SARS, and H1N1 pandemics. Earnest and protracted attempts at ECMO therapy in the anticipation of recovery should be balanced by clinical prudence that recognizes medical futility and allows for discontinuation of ECMO at an opportune time. Attempts at engaging family members early on regarding limitations and complications of ECMO and thresholds of futility are recommended.

\section{SUMMARY}

ECMO is a resource-intensive therapy that can be considered in highly selected patients. Expert centers should employ an evidence-based ARDS treatment algorithm and a multidisciplinary approach to recommending ECMO upon failure of conventional therapy. However, in resource-poor environments, the priority should be on ensuring essential respiratory support and the use of personal protective equipment to prevent morbidity and the spread of COVID-19. When ECMO is instituted, it should be offered to those who have the highest chance of survival.

Provisions should be made for adequate infection control precautions and the safety of the healthcare workers caring for ECMO patients.

Institutional investment in ECMO therapy should not divert resources away from basic medical and critical care.

Although the high mortality rate despite ECMO support cannot be discounted, its consideration in a highly selected cohort may be promising, given prior experiences with similar respiratory epidemics in the past.

\section{REFERENCES}

1. Guan WJ, Ni ZY, Hu Y, et al. Clinical characteristics of coronavirus disease 2019 in China. N Engl J Med 2020 Feb 28;NEJMoa2002032. doi:10.1056/NEJMoa2002032

2. Xu Z, Shi L, Wang Y, et al. Pathological findings of COVID-19 associated with acute respiratory distress syndrome. Lancet Respir Med 2020; 8(4):420-422. doi:10.1016/\$2213-2600(20)30076-X

3. Davies A, Jones D, Bailey M, et al. Extracorporeal membrane oxygenation for 2009 influenza $A(H 1 N 1)$ acute respiratory distress syndrome. JAMA 2009; 302(17):1888-1895. doi:10.1001/jama.2009.1535

4. Alshahrani MS, Sindi A, Alshamsi F, et al. Extracorporeal membrane oxygenation for severe Middle East respiratory syndrome coronavirus. Ann Intensive Care 2018; 8(1):3. doi:10.1186/s13613-017-0350-x

5. Combes A, Hajage D, Capellier G, et al. Extracorporeal membrane oxygenation for severe acute respiratory distress syndrome. N Engl J Med 2018; 378(21):1965-1975. doi:10.1056/NEJMoa1800385

6. Ñamendys-Silva SA. ECMO for ARDS due to COVID-19 (Letter) Heart Lung 2020 Mar 26;S0147-9563(20)30100-X. doi:10.1016/j. hrtlng.2020.03.012

7. 21 of 40 critical coronavirus patients in Japan recovered on ECMO life support. The Mainichi April 3, 2020. https://mainichi.jp/english/ articles/20200403/p2a/00m/0na/010000c. Accessed April 27, 2020.

8. Grasselli G, Zangrillo A, Zanella A, et al. Baseline characteristics
TABLE 1

Indications for and contraindications to venovenous extracorporeal membrane oxygenation (ECMO) in COVID-19

ECMO centers should develop individual center-specific inclusion and exclusion criteria and consider patients on a case-by-case basis

\section{Indications}

Severe acute respiratory distress syndrome with $\mathrm{PaO}_{2}: \mathrm{FiO}_{2}$ ratio $<100$ despite high positive end-expiratory pressure $\left(8-10 \mathrm{~cm} \mathrm{H}_{2} \mathrm{O}\right)$ and $\mathrm{FiO}_{2}>80 \%$

Trial of paralytics and prone positioning attempted

Absolute contraindications

Advanced age

Clinical Frailty Scale category $\geq 3$

Terminal disease with short expected survival

End-stage malignancy

Severe neurologic damage

Severe multiorgan failure

Mechanical ventilation $>10$ days

Unable to accept blood products

Contraindications to anticoagulation

Do-not-resuscitate status

Relative contraindications

Age $\geq 65$

Obesity (body mass index $\geq 40 \mathrm{~kg} / \mathrm{m}^{2}$ )

Multiple comorbid conditions

Severe immunosuppression

Septic shock

and outcomes of 1591 patients infected with SARS-CoV-2 admitted to ICUs of the Lombardy region, Italy. JAMA Apr 6;e205394. doi:10.1001/jama.2020.5394

9. Shi S, Qin M, Shen B, et al. Association of cardiac injury with mortality in hospitalized patients with COVID-19 in Wuhan, China. JAMA Cardiol 2020 Mar 25;e200950. doi:10.1001/jamacardio.2020.0950

10. Li X, Guo Z, Li B, et al. Extracorporeal membrane oxygenation for coronavirus disease 2019 in Shanghai, China. ASAIO J 2020 Mar 30 doi:10.1097/MAT.0000000000001172

11. Tang N, Li D, Wang X, Sun Z. Abnormal coagulation parameters are associated with poor prognosis in patients with novel coronavirus pneumonia. J Thromb Haemost 2020; 18(4):844-847. doi:10.1111/jth.14768

12. World Health Organization. Clinical management of severe acute respiratory infection (SARI) when COVID19 disease is suspected. Geneva: World Health Organization; 2020 Mar 13. https://www. who.int/publications-detail/clinical-management-of-severe-acuterespiratory-infection-when-novel-coronavirus-(ncov)-infection-issuspected. Accessed 24 April 2020.

13. Bartlett RH, Ogino MT, Brodie D, et al. Initial ELSO guidance document: ECMO for COVID-19 patients with severe cardiopulmonary failure. ASAIO J 2020 Mar 30. doi:10.1097/MAT.0000000000001173 